\title{
Neural Layer Bypassing Network
}

This paper was downloaded from TechRxiv (https://www.techrxiv.org).

\section{LICENSE}

CC BY 4.0

SUBMISSION DATE / POSTED DATE

$13-10-2021 / 20-10-2021$

CITATION

Palasamudram, Amogh (2021): Neural Layer Bypassing Network. TechRxiv. Preprint. https://doi.org/10.36227/techrxiv.16806928.v1

$\mathrm{DOI}$

10.36227/techrxiv.16806928.v1 


\title{
Neural Layer Bypassing Network
}

\author{
A Novel Architecture to Increase the Speed of Forward \\ Propagation without Compromising on Accuracy, Network \\ Structure, or CPU Load
}

By: Amogh Palasamudram

Mentor: Dr. Ronjon Nag

\section{Abstract}

This research aims to introduce and evaluate a new neural network architecture to improve the speed and effectiveness of forward propagation in neural networks: the Neural Layer Bypassing Network (NLBN). The theory and workings of this architecture have been explained in this research paper, along with comparisons to other methods of increasing the efficacy of deep learning models. This research also includes code examples with 3 image classification models trained on different datasets and analyses the impact of the NLBN architecture on forward propagation. It was found that this architecture increases the speed of forward propagation and tends to slightly decrease the accuracy of the model. However, it takes longer to train and takes more memory. All in all, this architecture is a potential foundation for using deep learning to teach neural networks to be more efficient. This includes skipping and re-propagating through layers to improve the overall performance of a model. 


\section{Introduction}

Neural Networks have significantly improved and dominated deep learning for decades. They can reach high levels of precision and recall and in some cases, outperform humans as well. One main reason why neural networks do well is because of their large and complex architectures. Though these architectures increase the accuracy of neural networks, they tend to take more time for forward propagation, so they output results slower than other deep learning architectures. This raises issues in real-time applications of neural networks, for instance, in self-driving cars, where a minuscule delay can create life or death situations for passengers.

Some have attempted to reduce the forward propagation time for deep neural networks. However, the majority of people solve this issue by reducing the number of layers or number of neurons per layer, which can improve prediction time, but this significantly decreases the accuracy and capability of neural networks due to potential underfitting. There are also certain architectures that help speed up deep learning applications without sacrificing the accuracy of the algorithm in various ways, but these solutions generally make use of different architectures, such as the 2017 Illustrated Transformer architecture. $^{1}$

In this research, I will be proposing a new architecture that uses the fundamental building blocks of standard neural networks, such as convolutional and pooling layers in the case of CNNs, to forward propagate and perform a task. However, I intend to have checkpoints after each layer to determine whether the forward propagation should continue or not. This decision depends on the probability or confidence of the model that a certain output should be produced after passing through a variable number of layers in the network.

\footnotetext{
${ }^{1}$ Attention is All You Need - 07/2021: https://arxiv.org/pdf/1706.03762.pdf
} 


\section{Related Work}

\subsection{Transformers}

Transformers are architectures that use self-attention to learn sequences across elements of inputs, allowing long-term dependencies and scalability. ${ }^{2}$ Initially they were used in Natural Language Processing but have been adapted to work in Computer Vision through data manipulation as well. ${ }^{3}$ Transformers provide attention and processing power to specific parts of the inputs that are important when obtaining the final output. This makes them more efficient as the network focuses on particular portions of the inputs rather than the entire input.

\subsection{Pipelining}

Machine Learning Pipelining is a technique to optimize the input data for a neural network to reduce the processing time and memory required for a model to perform forward

\footnotetext{
2 Transformers in Vision: A Survey - 10/2021: https://arxiv.org/pdf/2101.01169.pdf

${ }^{3}$ Transformers in CV - 10/2021: https://towardsdatascience.com/transformer-in-c v-bbdb58bf335e
}

propagation. ${ }^{4}$ This ranges from preprocessing and compressing data for neural networks to use. Some examples include Processor Pipelining and Instruction Pipelining.

\section{Architecture}

I am proposing a new architecture (the Neural Layer Bypassing Network or NLBN) that revolves around the standard neural network structure but adds a new layer (the rejection layer) to each layer of the main model to determine the probability of the semi-processed input resulting in the expected output. If this probability is below a trainable or fixed threshold, the forward propagation will be stopped and the next input will be taken for forward propagation.

The rejection nodes are not expected to have the precision and recall of a complete neural network. They are present to determine whether there is

\footnotetext{
${ }^{4}$ Entropy-Aware I/O Pipelining for Large-Scale Deep Learning on HPC Systems - 10/2021 https://ieeexplore.ieee.org/abstract/document/85 $\underline{26881}$
} 
any possibility that the inputs contain the expected results. If a certain outcome is extremely likely, based on the results of the preceding layers, the rejection layers will stop forward propagation and move on to the next input. Else, if there is some chance that the expected result is present within this semi-processed data, the forward propagation will continue.

It is expected that the thresholds for the rejection layers will increase as the layer number increases.

Figure 1 - Illustration that depicts how this architecture works in a standard convolutional neural network.

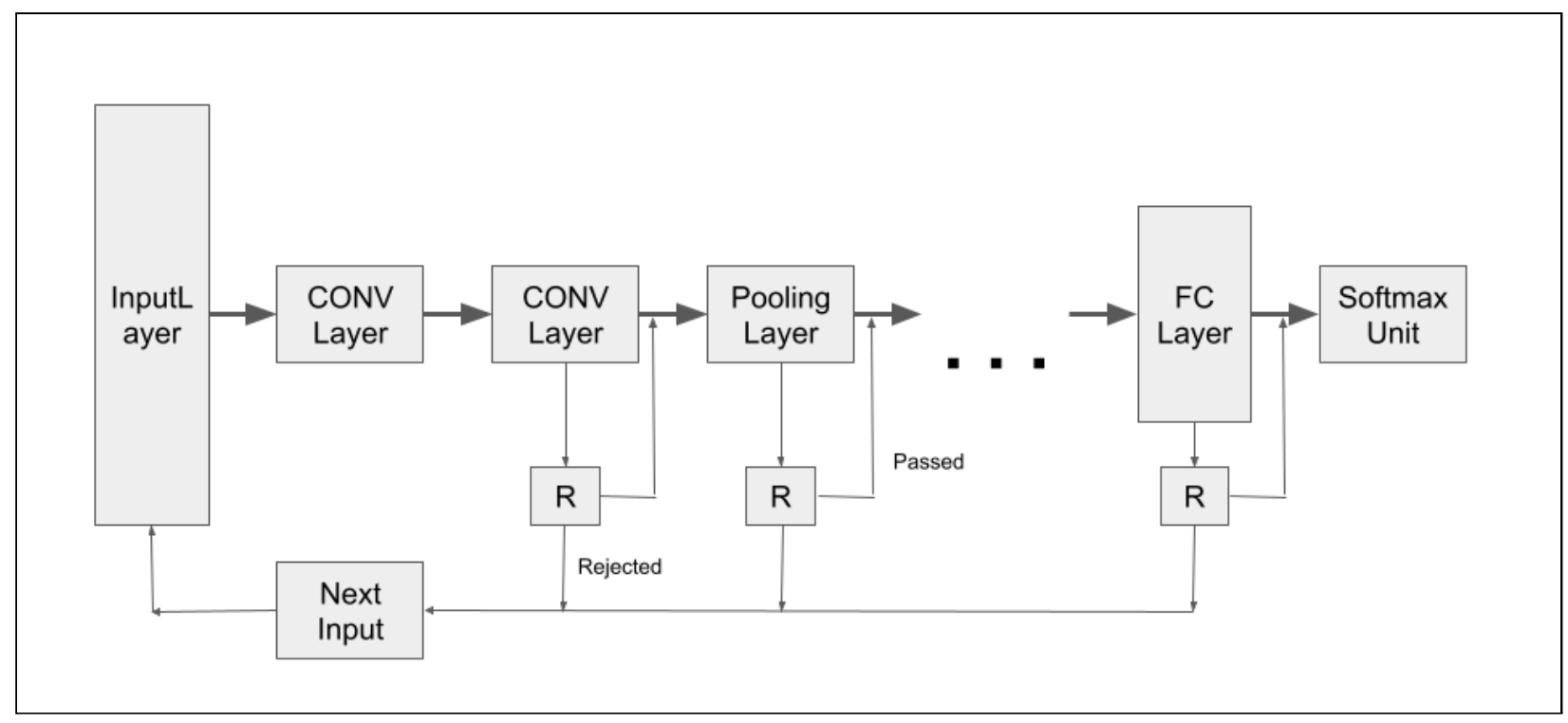

In Figure 1, the Legend is as follows:

1. CONV Layer - Convolutional Layer for Feature Extraction.

2. Pooling Layer - Average or Max Pooling Layer for Feature Extraction.

3. FC Layer - Fully Connected Layer with standard weights for classification.

4. $\mathbf{R}$ - Rejection Layers to reject input if it is unlikely to have an expected result.

This approach to speed up neural networks is mostly applicable for networks used for real-time classification problems or utilities that require less power consumption and faster predictions. This includes public chatbots with millions of simultaneous users and smart security cameras that alert owners when they detect an unidentified individual on their property. 
This architecture is expected to reduce the number of inputs that must fully propagate through the neural network, hence, reducing the average number of computations and the average prediction time per input. With this in mind, a possible drawback is an increase in training time and memory required due to the extra rejection layers. Additionally, the recall or precision of the network may decrease depending on the number of layers and labels, since inputs can be rejected at any stage of the network, potentially increasing the false negatives or increasing the false positives from predictions.

\section{Experiments}

To determine the effectiveness of the NLBN architecture I have proposed, I will be using Google COLAB to analyze the performance of my model. I have coded a COLAB Jupyter Notebook with the NLBN architecture to compare with an identical Convolutional Neural Network (CNN), the Standard Model, without the rejection layers. This was created using the Keras ${ }^{5}$ and

\footnotetext{
${ }^{5}$ Keras API - 07/2021: https://keras.io/api/
}

TensorFlow ${ }^{6}$ open source libraries. Additionally, this research will be evaluating the accuracy and forward propagation speed of the different architectures because the differences in these architectures directly influenced these properties of the models. Moreover, I will be analyzing the difference in the training accuracy since hyperparameterization such as regularization is out of the scope of this research.

I will be using 3 different datasets for this experimentation: MNIST Handwritten Digits Dataset, Horses or Humans Dataset, and the Colorectal Histology Dataset. These datasets are provided by Tensorflow, ${ }^{7}$ and have a wide variety of features, including but not limited to image type, image size, number of images, and difficulty in classifying images. This variety will allow me to more accurately determine the effectiveness of my new NLBN model architecture.

\footnotetext{
${ }^{6}$ TensorFlow Libraries - 07/2021: https://www.tensorflow.org/resources/libraries-ex tensions

${ }^{7}$ TensorFlow Datasets - 07/2021: https://www.tensorflow.org/datasets/catalog/
} 


\subsection{Creating the NLBN Model}

To create the Neural Layer Bypassing Network, I performed the following steps which can be seen in the linked code:

1. Import the necessary packages and libraries.

2. Upload and preprocess the training and testing data (in this case images).

3. Create a list of TensorFlow / Keras layers that would succeed one another in a model.

4. Create a list of TensorFlow / Keras models (Broken Models bModels) that input the layer from the previous index and outputs the layer in the same index.

a. The first index (0) will input the images from the training data.

5. Train the last model in the Broken Models list; this will automatically train the preceding models.

6. Create a list of rejection layers (Rejection Models - rModels) that input the layer in the same index of the Broken Models and outputs the probability of an image being a certain class.

7. Train each rejection model.
8. Create the final list of models that are used for predictions (Structured Models - sModels). Use the layers in the layers list created earlier and load the weights of the respective layers in the Broken Models list into these layers.

9. Create a list of confidence levels to determine when to exit forward propagation.

10. Forward propagate through the Structured Models:

a. Forward propagate through the first structured model.

b. Forward propagate through the first rejection layer.

c. Compare the maximum value of the prediction to the assigned confidence level of the specific layer. If the value is greater, exit forward propagation with this label. Else, resume forward propagation in the next layer.

d. Repeat the previous step until the NLBN model exits forward propagation or passes through the entire model. 


\subsection{Predictions on MNIST}

\section{Handwritten Digits Dataset}

For a preliminary test, I used the MNIST Handwritten Digits Dataset. ${ }^{8}$ This dataset contains $28 \times 28$ pixel images of the digits 0 to 9 with an even distribution. The architecture I used can be seen in Figure 2.

Figure 2 - Diagram of CNN Architecture for MNIST dataset.

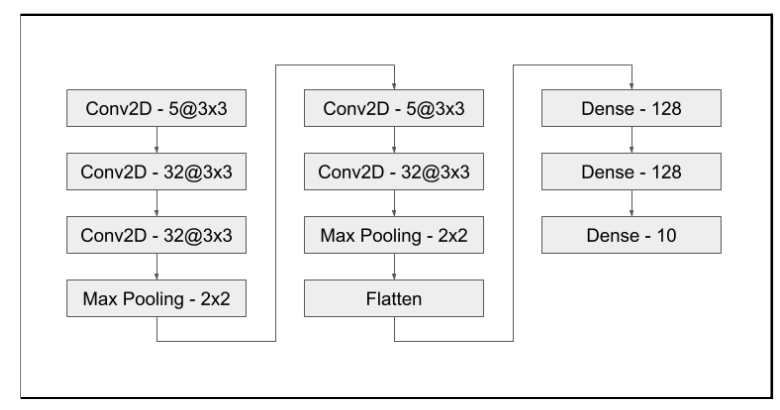

After training the NLBN and the standard model using CONV2D and Pooling layers from the Keras libraries, I tested them on the 60,000 images in this dataset. This was done in the specific COLAB notebook. ${ }^{9}$ I found the accuracy of the standard CNN to be $99.2 \%$; the time for its forward

\footnotetext{
${ }^{8}$ MNIST Handwritten Digits Dataset - 07/2021: https://web.stanford.edu/ hastie/CASI files/DAT A/MNIST.html

${ }^{9}$ Neural Layer Bypassing Network (NLBN) MNIST Digits Dataset: https://colab.research.google.com/drive/1KiFhm Rnmuasj oTUNWmncqWiuGkOOsSo?usp=shar ing
}

propagation for all 60,000 examples was 2340 seconds. The accuracy for the NLBN architecture was $95.8 \%$; the time taken for forward propagation for all 60,000 examples was 2160 seconds.

Overall, the accuracy decreased by $3.4 \%$, while the time taken for forward propagation decreased by $7.7 \%$, meaning that the speed of forward propagation increased by $8.3 \%$. It can be inferred that this speed increased because the NLBN model did not pass all the images through the entire network, resulting in fewer computational steps, and the accuracy reduced as a result of the reduction in the recall and precision due to the rejection layers in the NLBN architecture.

The results of this experiment suggest a small difference between the performance of the NLBN versus the standard CNN architecture. This can be attributed to the dataset not being completely applicable for the NLBN's use case. The MNIST Handwritten Digits Dataset is known to be easy to train a model with fewer layers on while attaining $+98 \%$ accuracy. To verify and 
determine the effectiveness of the NLBN model, I will also be using the 2 other datasets mentioned earlier.

\subsection{Predictions on Horses or} Humans Dataset

For another, basic analysis, I used the horses_or_humans dataset, offered by Google COLAB. ${ }^{10}$ This dataset has 1000+ images and a 50:50 distribution of horse and human pictures with a resolution of $300 \times 300$ in RGB. The architecture I used can be seen in Figure 3. I used a different COLAB notebook to test this dataset. ${ }^{11}$

Figure 3 - Diagram of CNN Architecture for Horses or Humans dataset.

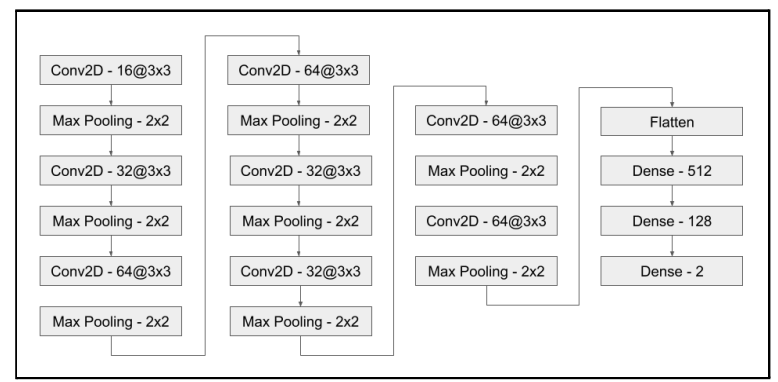

\footnotetext{
${ }^{10}$ Horses or Humans Dataset - 07/2021: https://www.tensorflow.org/datasets/catalog/hors es or humans

${ }^{11}$ Neural Layer Bypassing Network (NLBN) Horses or Humans Dataset: https://colab.research.google.com/drive/1SEvW YxPNvFXx163PdvPADS4L7iogcxN?usp=shari ng
}

After training this NLBN model on this dataset, I tested the NLBN and standard models on the training set. The accuracy for the standard model was $100 \%$, while the total time taken was 126 seconds for the whole training dataset. However, the accuracy for the NLBN model was $100 \%$ as well. The total time taken to predict all the training images was only 91 seconds. The new architecture reduced the forward propagation time by $27.8 \%$ and increased the forward propagation speed by $38.5 \%$, keeping the accuracy constant.

Because there are only 2 classes, humans and horses, which are easily differentiable, it is possible that the models found it significantly easier to classify images using fewer layers, compared to the data in the MNIST dataset. As a result, the model obtains $100 \%$ accuracy. The fact that this accuracy did not decrease for the NLBN model suggests that, generally, for most of the training data, only a few layers were required to determine the label of the image with high precision and recall. This resulted in the NLBN exiting forward propagation early, before 
passing through the entire network, thus saving time and increasing the average speed of classifying predictions without affecting accuracy.

The NLBN model has shown significant improvement in forward propagation speed without sacrificing the accuracy of the model through a potential drop in the recall. To further highlight the impact of this new architecture, I will apply it on one last dataset.

\subsection{Predictions on Colorectal}

\section{Histology Dataset}

For the final dataset, I used the colorectal_histology dataset, provided in the TensorFlow Datasets package. ${ }^{12}$ I also used a new COLAB notebook to test the NLBN model on this dataset. ${ }^{13}$ There are 8 evenly distributed classes in the dataset, which has 5,000 examples; the images are in RGB with the resolution 150x150 and have been split 80:20 for training and testing. Figure 4 depicts the architecture I have used.

\footnotetext{
${ }^{12}$ Colorectal Histology Dataset - 07/2021: https://www.tensorflow.org/datasets/catalog/colo rectal histology

${ }^{13}$ Neural Layer Bypassing Network (NLBN) Colorectal Histology Dataset:

https://colab.research.google.com/drive/17ml4X kn01lle5XoSt0oAdCVJ2O1DiX2y?usp=sharing
}

Figure 4 - Diagram of CNN Architecture for Colorectal Histology dataset.

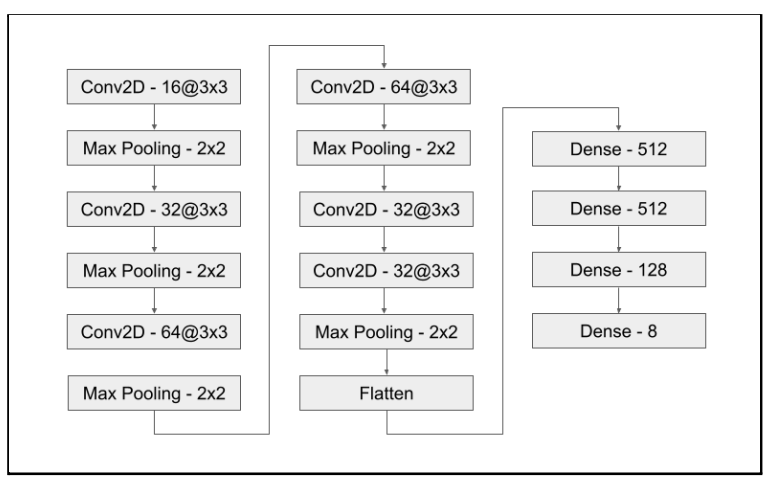

After training this NLBN model on this colorectal histology dataset, I tested the NLBN and standard models on the training data. The accuracy of the standard model was $97.1 \%$, and the total time taken to predict all training examples was 224 seconds for the whole training dataset. However, the accuracy for the NLBN model was $94.8 \%$, and the total time taken to predict all the training images was only 200 seconds. The new architecture reduced the forward propagation time by $10.7 \%$ and increased the forward propagation speed by $12.0 \%$ while reducing the accuracy by $2.3 \%$.

The increase in speed of forward propagation can again be attributed to the reduced number of layers required to make accurate predictions. However, 
the $12.0 \%$ increase in speed of forwards propagation is less than the increase in speed for the Horses or Humans dataset, possibly because the Colorectal Histology dataset contains images of cells, which are more complex, intricate, and generally difficult to classify.

Moreover, the accuracy as expected decreased. This is most likely due to the reduction in recall and precision due to the high confidence levels and less accurate predictions of the rejection layers. However, the accuracy decrease is less than that of the NLBN model trained on the MNIST dataset, despite the increase in speed being greater for the Colorectal Histology dataset. This can potentially be attributed to a mild case of vanishing and exploding gradients in the standard model due to the presence of an excess number of layers, which slightly hindered training in comparison to the model trained on the MNIST dataset. However, the rejection layers are trained with a different number of preceding layers, preventing the NLBN model from experiencing the vanishing and exploding gradient problem.
The NLBN model has improved the speed of forward propagation though it slightly decreased the accuracy of the model through a potential avoidance of the vanishing and exploding gradients problem.

\subsection{Graphical Analysis}

For each of the models trained on the 3 different datasets, I have recorded the $\%$ decrease in accuracy, \% increase in forward propagation speed, and $\%$ decrease in time per prediction between the standard and NLBN models. The graphical representation can be seen in Figure 5.

Figure 5 - Bar Chart of the \% change in different performance measures between the Standard model and the NLBN model.

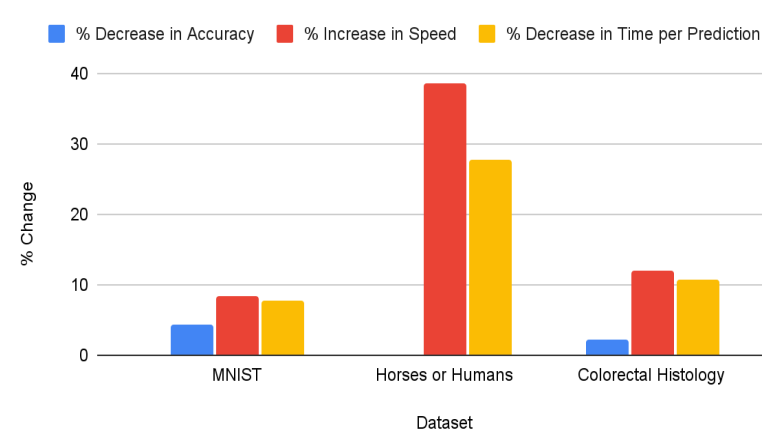

In general, the $\%$ decrease in accuracy is significantly less than the $\%$ decrease in time per prediction. Moreover, the \% increase in speed is always greater than the $\%$ decrease in time per prediction. 
These trends suggest that the NLBN architecture improves forward propagation speed more than it harms the accuracy. However, the degree of efficacy depends on the application of the network.

\section{Conclusion}

The NLBN model architecture reduces the time taken for forward propagation per image or training example. This results in an increase in the propagation speed as the models tend to exit forward propagation at earlier layers, reducing the number of computations.

The accuracy tends to decrease, due to the use of rejection layers to exit forward propagation midway which could possibly reduce recall and precision.

This is because labels may be predicted inaccurately in rejection layers with high confidence, thus increasing the number of false positives and false negatives predicted by the NLBN model.

Additionally, the degree by which the accuracy reduces for different NLBN models and datasets may vary due to the vanishing and exploding gradients issue. There can be an ideal number of layers for predicting examples from the dataset without facing the vanishing and exploding gradient issue and avoiding underfitting simultaneously. If an NLBN network is used, it can capitalize on the ideal number of layers, assuming this is less than the total number of layers in the model, hence, reducing the decrease in accuracy relative to other models.

Overall, the NLBN tends to increase the forward propagation time, and will most likely decrease accuracy by a percentage relatively less than the percentage increase in speed. The exact results heavily depend on the application, model structure, and dataset. The effectiveness of the NBLN can be determined by the significance of each feature of the model; these features include precision, recall, speed, CPU load, and more.

\section{Further Scope}

\section{Further Improvements}

This research delves into the theoretical and preliminary implementation of this newly proposed NLBN model architecture. It has been implemented in 
Google COLAB using TensorFlow and Keras packages. Because it is new, the TensorFlow and Keras libraries are not highly efficient for the application of the NLBN architecture, considering the abundance of if-statements and the lack of matrix multiplications for the coded prediction method.

Furthermore, the confidence levels for the rejection layers were fixed, rather than trained. If they were trained, it is likely the accuracy, training speed, and forward propagation speed would increase.

Finally, this NLBN model architecture can be further improved through more investigation and can be deployed in more applicable use cases, such as in smart security cameras or in autonomous vehicles.

\section{Potential \& Future Applications}

This research ventures into the beginnings of deep learning models that do not follow the traditional method of forward propagating through all the layers in a model. This NLBN model lays the ground for other potential neural network architectures that may learn to skip or propagate through layers to improve performance in terms of accuracy, CPU load, and speed. In a more intuitive sense, the next steps are to teach deep learning models to be more effective and efficient through deep learning. Analogous to human perception, this idea is similar to humans thinking and understanding their thought process and learning how to think in the future.

Though this NLBN architecture requires more memory and more time to train, because it has extra rejection layers; the impact of these is based on the resources, circumstances, and limitations of developers for the given applications. 


\section{Acknowledgements \& References}

I would like to thank Dr. Ronjon Nag for supporting me and guiding me through this research.

[1] Attention is All You Need - 07/2021: https://arxiv.org/pdf/1706.03762.pdf

[2] Transformers in Vision: A Survey 10/2021:

https://arxiv.org/pdf/2101.01169.pdf

[3] Transformers in CV - 10/2021:

https://towardsdatascience.com/transformer -in-cv-bbdb58bf335e

[4] Entropy-Aware I/O Pipelining for Large-Scale Deep Learning on HPC Systems - 10/2021

https://ieeexplore.ieee.org/abstract/docume nt/8526881

[5] Keras API - 07/2021: https://keras.io/api/

[6] TensorFlow Libraries - 07/2021:

https://www.tensorflow.org/resources/librarie $\underline{\text { s-extensions }}$

[7] Tensorflow Datasets - 07/2021:

https://www.tensorflow.org/datasets/catalog/
[8] MNIST Handwritten Digits Dataset 07/2021:

https://web.stanford.edu/ hastie/CASI files/ DATA/MNIST.html

[9] Neural Layer Bypassing Network (NLBN) - MNIST Digits Dataset:

https://colab.research.google.com/drive/1Ki FhmRnmuasj oTUNWmncqWiuGkOOsSO? usp=sharing

[10] Horses or Humans Dataset - 07/2021: https://www.tensorflow.org/datasets/catalog/ horses or humans

[11] Neural Layer Bypassing Network (NLBN) - Horses or Humans Dataset: https://colab.research.google.com/drive/1SE vW YxPNvFXx163PdvPADS4L7iogcxN?us $\mathrm{p}=$ sharing

[12] Colorectal Histology Dataset - 07/2021: https://www.tensorflow.org/datasets/catalog/ colorectal_histology

[13] Neural Layer Bypassing Network (NLBN) - Colorectal Histology Dataset: https://colab.research.google.com/drive/17 ml4Xkn01lle5XoSt0oAdCVJ2O1DiX2y?usp sharing 